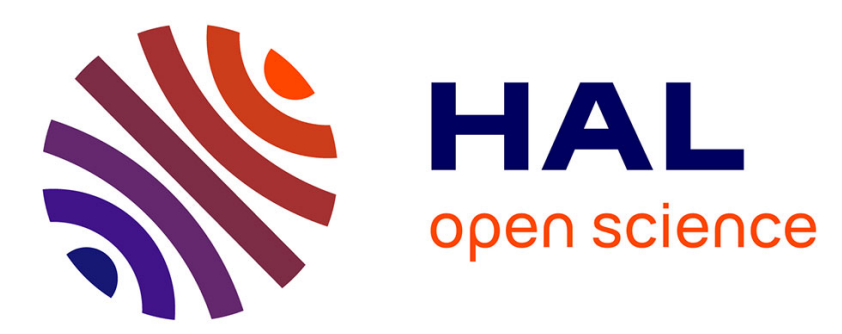

\title{
Mapping acoustic field distributions of VHF to SHF SAW transducers using a Scanning Electron Microscope.
}

Aurélien Godet, Jean Friedt, Sounkalo Dembele, Nadine Piat, Abdelkrim

Khelif, Pascal Vairac, Joel Agnus, Pierre Bourgeois, Gwenhaël Merou

\section{> To cite this version:}

Aurélien Godet, Jean Friedt, Sounkalo Dembele, Nadine Piat, Abdelkrim Khelif, et al.. Mapping acoustic field distributions of VHF to SHF SAW transducers using a Scanning Electron Microscope.. European Frequency and Time Forum, Apr 2018, Turin, Italy. hal-02380406

\section{HAL Id: hal-02380406 https://hal.science/hal-02380406}

Submitted on 26 Nov 2019

HAL is a multi-disciplinary open access archive for the deposit and dissemination of scientific research documents, whether they are published or not. The documents may come from teaching and research institutions in France or abroad, or from public or private research centers.
L'archive ouverte pluridisciplinaire HAL, est destinée au dépôt et à la diffusion de documents scientifiques de niveau recherche, publiés ou non, émanant des établissements d'enseignement et de recherche français ou étrangers, des laboratoires publics ou privés. 


\title{
Mapping acoustic field distributions of VHF to SHF SAW transducers using a Scanning Electron Microscope
}

\author{
A. Godet, J.M. Friedt, S. Dembélé, N. Piat, A. Khelif, P. Vairac, J. Agnus, P.Y. Bourgeois, G. Goavec-Mérou \\ FEMTO-ST Institute UMR 6174, Université de Franche-Comté, CNRS, ENSMM, UTBM \\ 26 rue de l'Épitaphe, 25000 Besançon, France
}

\begin{abstract}
Mapping the energy distribution of Surface Acoustic Wave (SAW) devices operating in the Very High Frequency (VHF) and Super-High Frequency $(\mathrm{SHF})$ range provides a quantitative indicator of energy confinement, a core parameter when addressing low loss filters or high quality factor resonators. We here demonstrate the use of Scanning Electron Microscopy (SEM) for mapping Rayleigh wave acoustic field and shear transverse wave (STW) propagating on quartz. Furthermore, the availability of Focused Ion Beam (FIB) for milling the piezoelectric substrate allows for creating obstacles on the acoustic path and hence tune the acoustic wave propagation direction by reflecting the waves along directions which might otherwise exhibit poor electromechanical coupling.
\end{abstract}

\section{CONTEXT AND MOTIVATION}

Acoustic field distribution in surface acoustic wave (SAW) devices relates to acoustic energy confinement and hence acoustic losses (in filters and delay lines) or quality factor (in resonators). Classical mapping techniques are based on optical interferometry [1], [2], [3], in which crystalline lattice motion associated with SAW propagation is detected in an interferometer setup with the SAW surface acting as one of the arm end. Despite the ability to quantitatively measure the out-of-plane vibration amplitude, optical interferometric methods are unable to measure in-plane vibration components. One competing approach is the observation of the electric field associated with SAW propagation in piezoelectric substrates [4], [5], [6], [7]: in scanning electron microscopy (SEM) observations, electrons illuminate the surface under investigation and secondary electrons generated closest to the surface are collected to create an image representative of surface characteristics. Electric fields on the surface under investigation modulate the secondary electron path and hence the image observed: SAW propagating is observed using SEM.

In this presentation, we use a SEM for the observation of Rayleigh SAW on lithium niobate and shear transverse waves (STW) propagating on quartz. In all cases, we focus on delay line geometries: despite not exhibiting a standing wave pattern as observed on resonators, the propagating wave is readily observed in both configurations. The reason for selecting these two experimental setups as appropriate to emphasize some advantages of the SEM approach over the optical characterization methods are in the former case the wavelength of the device - operating at $2.45 \mathrm{GHz}$ with an acoustic velocity of $3992 \mathrm{~m} / \mathrm{s}$ [8] - exhibits a wavelength of $1.6 \mu \mathrm{m}$ or only 2 to 5 optical wavelengths, and in the latter case the shear polarization of the wave which does not exhibit out-of-plane displacement component. In all cases, we have also observed that SEM imaging speed - a few seconds at most - is greatly improved over the raster scanning technique of the optical interferometer which always last a few minutes to hours : a $1024 \times 768$ pixel SEM image requires an acquisition time of $122 \mathrm{~ms}$, allowing much faster sampling rates than scanning probe techniques lasting at best 20 minutes for $2500 \times 400$ pixels for the fastest instruments [9].

\section{HISTORICAL BACKGROUND}

SEM characterization has periodically reappeared in the history of acoustic device characterization. The oldest record we have identified of dynamic oscillator characterization using SEM dates back to 1969 [10], while the observation of the electric field associated with acoustic wave propagation in piezoelectric media dates back to 1971 [11] for bulk acoustic resonators and 1978 for SAW [12]. Interest has grown in this field until 1980, with afterwards a ten year gap until renewed interest sparked a series of publications in the 1990s. Finally, the SEM has become a standard tool for characterizing even exotic substrates such as langasite [13], yet most investigations focus on standing wave patterns. Despite the bandwidth limitation of the scintillator used to convert the electrons to an optical signal, dynamic phenomena monitoring require some bandwidth increase so that the instrument reaches a larger bandwidth than the time constant of the physical phenomena under investigation. The classical approach to this dilemma is stroboscopy, in which the slow detector is only illuminated while the physical phenomenon is in a known, repeatable state, by a short incoming signal. In the case of SEM, the illuminating electron beam is chopped in order to illuminate the SAW device only when the wave packet is in a known position, yielding such famous images as those found in [14].

One explanation provided for the ability to visualize propagating SAW using SEM, despite the lack of reflectors to create the standing wave pattern found in resonators, is an interference mechanism between the electromagnetic field radiated by the interdigitated transducers - propagating at the speed of light and virtually instantaneously with respect to the acoustic wave speed - and the electric field associated with the acoustic wave propagation on the piezoelectric media. Because 
these two fields are synchronous, the interference pattern is stationary as seen by the slow SEM detector.

\section{EXPERIMENTAL MEASUREMENTS}

All experiments have been performed in a Carl Zeiss Auriga 60 SEM fitted with a focused ion beam (FIB, $\mathrm{Ga}^{+}$ions accelerated at $30 \mathrm{kV}$ ) capability. The electron acceleration voltage was reduced to $1 \mathrm{kV}$ to reduce charge accumulation on the piezoelectric insulating surface since at the low magnification we will consider throughout this investigation, higher acceleration voltage would induce excessive static buildup with little improvement in resolution. Working distance was set to about $5.1 \mathrm{~mm}$ for the sample to be close to the convergence point of the FIB and SEM column axis. The signal of a secondary electron (SE) detector inside the electron column (in-lens) was used. A Rohde \& Schwarz SMC 100A synthesizer was used to drive the SAW devices.

\section{A. Imaging $2450 \mathrm{MHz}$ Rayleigh wave on lithium niobate}

$2450 \mathrm{MHz}$ acoustic delay lines were imaged using an SEM. In all cases, the radiofrequency power is $+15 \mathrm{dBm}$ and the device is continuously powered. In all images, the reference image of the bare surface is shown on the right side of the picture, and the powered device with the acoustic wave propagating on the substrate is shown on the left. Fig. 2 exhibits a zoom on the acoustic path visible in the broad view of Fig. 1, with the interdigitated transducer on the top and the first mirror on the bottom.
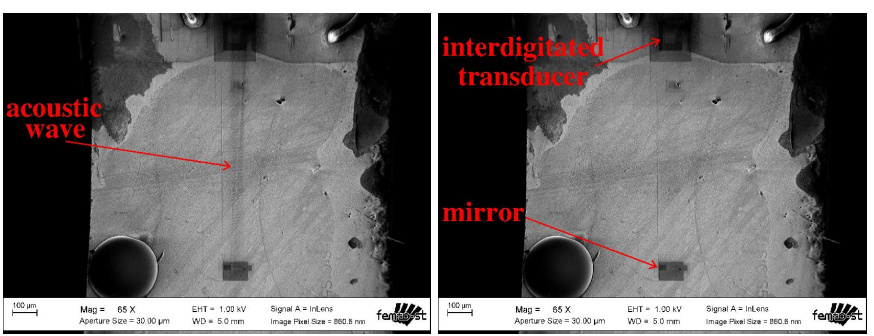

Fig. 1. General view of he acoustic delay line, with the interdigitated transducer on top and the first mirror on the bottom.

The collimation of the acoustic beam is well defined and the divergence is low enough for the acoustic field to be confined to the area over which the mirrors are located, even after a propagation path long enough to allow for a 500 ns delay.
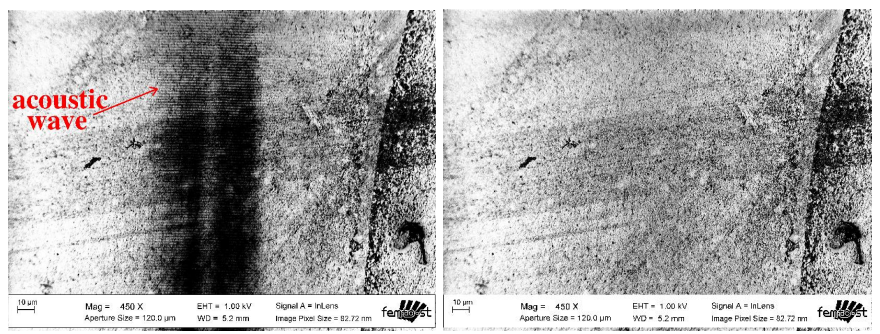

Fig. 2. Zoom on the acoustic path.

\section{B. Obstacles to $2450 \mathrm{MHz}$ Rayleigh waves}

In addition to observing the freely propagating Rayleigh SAW, obstacles were patterned using the FIB to either generate point-like source, or bounce the wave off-axis. Adding obstacles was on the one hand attempted to assess whether the milling depth is sufficient to affect a Rayleigh SAW wave, and create structures hardly accessible by patterning interdigitated transducers such as a point-like source (Fig. 3), propagation in any direction independently of the electromechanical coupling coefficient (Fig. 4) or even a half-coral (Figs. 5-7).
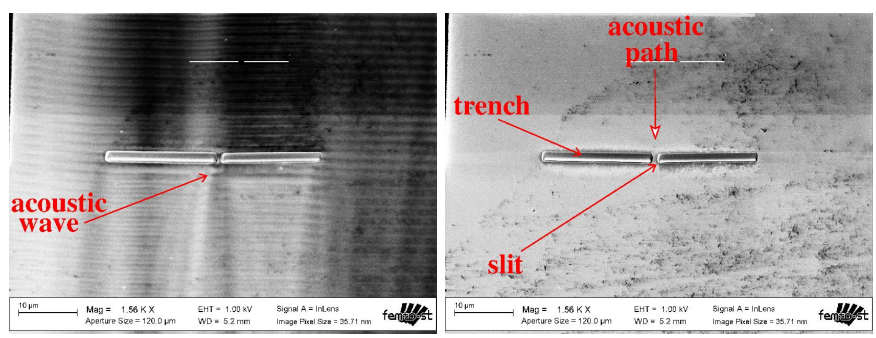

Fig. 3. A point-like source is created by patterning two trenches separated by a slit through which the acoustic field coming from the top scatters.

In the former example, the point like source is created by a introducing a slit between two obstacles with a width of the order of the wavelength. The scattered wave is well visible beyond the slit, on the bottom of Fig. 3 .
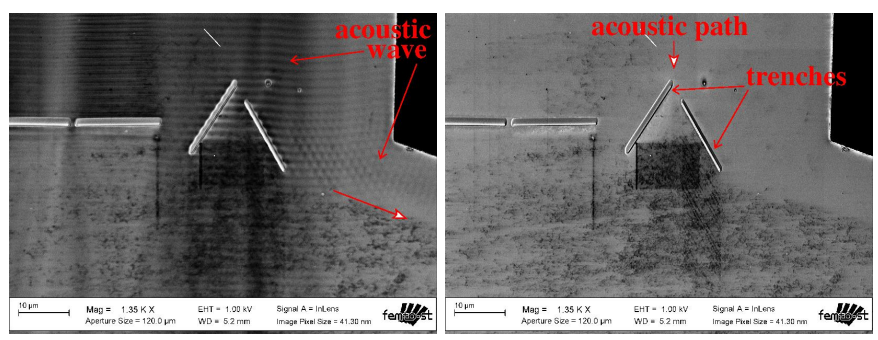

Fig. 4. Oblique trenches are patterned on the acoustic path: the trench deflecting the acoustic field towards the right exhibit strong efficiency and the deflected beam is well visible, while the trench supposed to deflect the beam towards the left has hardly any effect on the incoming wave.

An oblique obstacle is patterned on the acoustic path to reflect the wave in a direction in which no mirror is located and demonstrate that indeed the SEM is able to image a propagating wave. Here no cause of standing wave pattern could be due to obstacles located on the path after reflection. The reflected wave is well visible towards the right of Fig. 4, but not on the left.

Finally, the half coral geometry was introduced to try and create some interference pattern from confined acoustic waves. The interference between the incoming and reflected wave is well visible on Fig. 5, while the broader view from Fig. 6 emphasizes some acoustic field leakage below the wall and through the slits between adjacent walls, and the lowest magnification of Fig. 7 shows some of the diverging beams created by the off-axis reflection in the coral walls and propagation through the interdigitated transducer.

Hence, the combination of SEM and FIB seems ideal for real time observations of obstacles created along the acoustic 


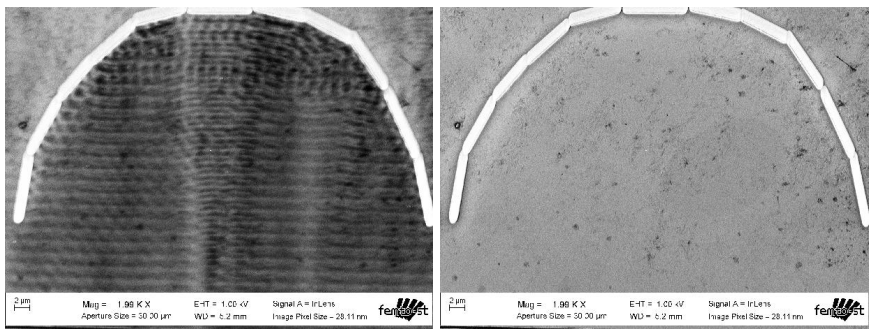

Fig. 5. Zoom on the half coral demonstrating the interference pattern between the incoming wave propagating from bottom to top and most significantly the wave reflected on the left oblique walls propagating towards the right of the image.
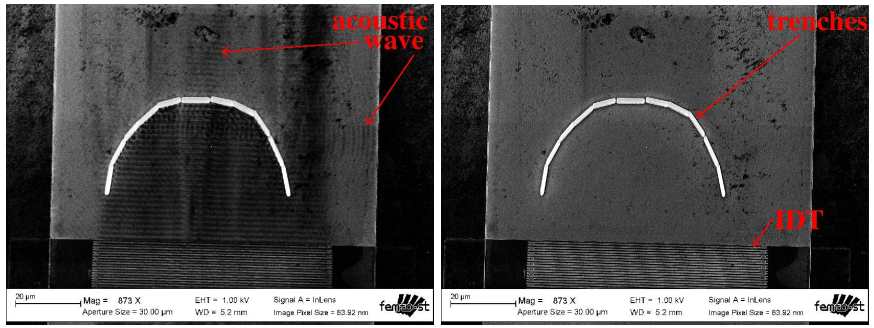

Fig. 6. The half coral lets some of the acoustic field escape under the trenches and through the slits connecting the trenches (top part of the left image), or some of the reflected energy leaks towards the right after propagating under the right-most trenches.

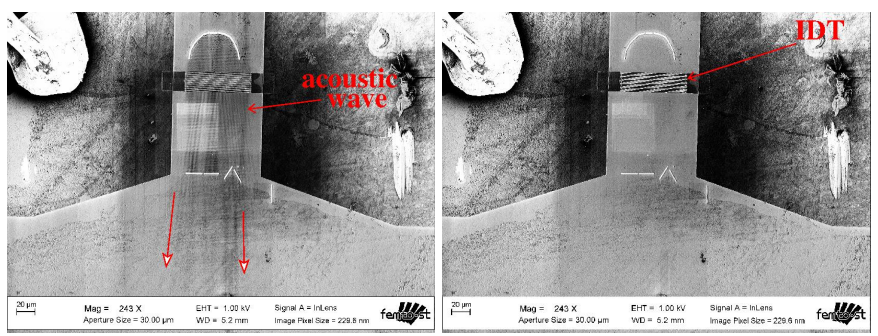

Fig. 7. Broad view of the SAW device with the half coral on the top region above the interdigitated transducers exhibiting the diverging acoustic beams on the acoustic path towards the mirrors on the bottom of the images.

path, and defining new propagation modes reminiscent to the principles used in phononic crystals. The high operating frequency is a mandatory condition for the shallow structures milled by the FIB to significantly affect the SAW propagation, as will be seen in the next section.

\section{C. $125 \mathrm{MHz}$ STW wave on quartz}

Observing Rayleigh wave propagation with a SEM on a strongly coupled substrate such as $\mathrm{YXI} / 128^{\circ}$ lithium niobate is well known and our contribution so far has been the addition of obstacles during the observation cycle. We are now interested in observing shear waves as the STW propagated on ST cut quartz, a measurement which is not possible using optical means due to the lack of out-of-plane vibration component.

The following pictures (Fig. 8) are taken as the delay line is powered by a continuous wave of $+18 \mathrm{dBm}$. The shear wave propagating beyond the electrodes in the area free of any structure on the left-side of the pictures is well visible. Here again, some obstacles were patterned on the acoustic path, with no effect this time. However, considering the shallow obstacles (the trench depth was measured using a mechanical profiler to be above $800 \mathrm{~nm}$ ) with respect to the wavelength, such a lack of reflection is hardly surprising.
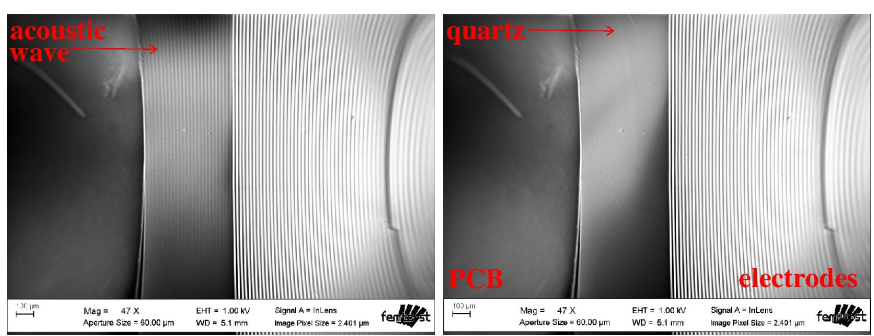

Fig. 8. STW wave propagating on quartz. The electrode pattern on the righ of the image connects the ground bus (towards the bottom of the image) to the top electrodes of the interdigitated transducers generating the wave (out of the field of these images). The area on the left of the electrode pattern is bare quartz free of metallic structures. The left-most part of the image is the printed circuit board on which the piezoelectric substrate is glued.

\section{DISCUSSION}

The observation of VHF to SHF SAW associated electric field should require a measurement bandwidth of at least twice the operating frequency. Measuring the transfer function of the secondary electron detector (Fig. 9) demonstrates a bandwidth of a few megahertz at most, suitable for microelectromechanical system characterization but well below the SAW operating frequency. Thus, the observation of a pattern representative of SAW propagation is either associated with a standing wave pattern (as found in resonator architectures), or in an interference between the radiated excitation signal and the electric field associated with SAW propagation on piezoelectric media [5].

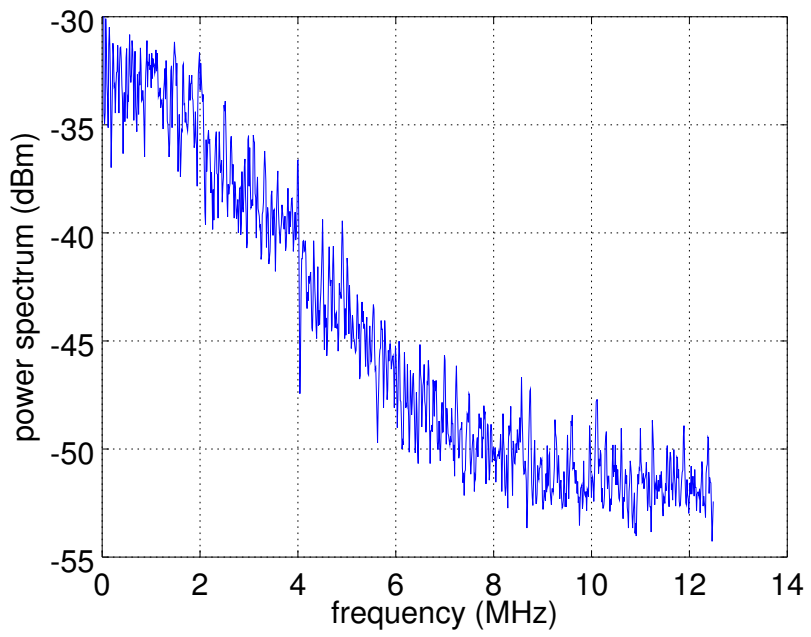

Fig. 9. SE2 secondary electron detector transfer function as observed from the noise power density spectrum.

The failure to observe a Love SAW confined in a $2 \mu \mathrm{m}$ silicon dioxide layer atop a quartz substrate hints at a strong interaction of the SEM electrons with the electric field at the surface of the piezoelectric material. Indeed, despite the electric field extending about a wavelength in air at the interdigitated electrode level $[8, \mathrm{p} .18]$, a thin $(5 \%$ of the acoustic wavelength) insulating layer prevents the secondary electrons from being affected by the electric field associated with acoustic wave propagation. 
Optical mapping of the STW device shown in Fig. 8 with the setup described in [15] indicates a $10 \mathrm{pm}$ vibration amplitude over the interdigitated transducers and negligible amplitude (below measurement resolution) in the bare quartz area, hence emphasizing the added value of SEM acoustic field mapping.

Finally, the operating frequency of the SAW device used for basic acoustic research in the context of interactions with the FIB-generated structure is dictated by the penetration depth of the wave in the substrate with respect to the ability of the trenches to significantly affect the acoustic wave velocity. The penetration depth of a Rayleigh wave in the piezoelectric substrate is of the order of the acoustic wavelength [16], while FIB-milled rectangles $72 \times 16 \mu \mathrm{m}^{2}$ at a current of $2 \mathrm{nA}$ in ST-cut quartz indicate depths of $18 \pm 1 \mathrm{~nm}, 52 \pm 1 \mathrm{~nm}$ and $128 \pm 2 \mathrm{~nm}$ for milling durations of $1.5,3$ and 6 minutes respectively. The resulting milling rate of $0.14 \pm 0.03 \mu \mathrm{m}^{3} / \mathrm{nC}$ is consistent with the values reported for lithium niobate in [17], [18]. Thus, trenches a few micrometers deep at most can be milled this way, and operating at high frequencies enhances the interactions of the wave with the structures. In our case, even after milling a trench for over 30 minutes at a current of $2 \mathrm{nA}$, no reflected wave was observed in the case of the $126 \mathrm{MHz}$ STW-wave propagating on quartz, while the effect was significant on the $2450 \mathrm{MHz}$ Rayleigh wave propagating on lithium niobate after milling durations of 60 seconds at a current of $200 \mathrm{pA}$, or 30 seconds for the half-coral shaped trenches.

\section{CONClusion}

We have repeated well known surface acoustic wave associated electric field mapping using scanning electron microscope, and extended the technique on the one hand to the observation of the influence of focused-ion beam induced structures on the acoustic path, and on the other hand to shear transverse wave propagation in quartz. While lacking the quantitative vibration amplitude characteristics of optical interferometric methods, the scanning electron microscope offers the ability of fast characterization and interaction with the acoustic field when a focused ion beam is available. Our experiments only use the low-bandwidth in-lens secondary electron detector for imaging standing wave patterns, whether resulting from reflections of the acoustic wave on Bragg mirrors or trenches patterned on the acoustic path, or resulting from the interference of the radiated electromagnetic field with the propagating acoustic wave. Further developments will focus on adding a stroboscopic imaging capability for time resolved acoustic wave packet imaging [19], [20], in which the slow detector is only illuminated by the incoming electron beam when the physical setup is in a known state defined by the excitation electric signal powering the interdigitated transducer powering the surface acoustic wave device.

\section{ACKNOWLEDGEMENT}

We acknowledge fruitful discussions with L. Reindl (IMTEK, Freiburg, Germany) who provided references on the historical background of SEM characterization of SAW devices. D. Teyssieux (FEMTO-ST, Besançon, France) proofread the manuscript and performed the optical analysis on the STWdevice. Funding for this project is provided by the (ANR11-LABX-01-01) ACTION Labex grant and supported by the (ANR-10-EQPX-44-01) ROBOTEX equipex as well as a 2015 ENSMM BQR grant.

\section{REFERENCES}

[1] J. V. Knuuttila, P. T. Tikka, and M. M. Salomaa, "Scanning michelson interferometer for imaging surface acoustic wave fields," Optics Letters, vol. 25, no. 9, pp. 613-615, May 2000.

[2] K. Kokkone and M. Kaivola, "Scanning heterodyne laser interferometer for phase-sensitive absolute-amplitude measurements of surface vibrations," Applied Physics Letters, vol. 92, no. 6, p. 063502, 2008.

[3] H. Martinussen, A. Aksnes, and H. Engan, "Wide frequency range measurements of absolute phase and amplitude of vibrations in microand nanostructures by optical interferometry," Optics express, vol. 15, no. 18, pp. 11370-11384, 2007.

[4] G. Eberharter and H. Feuerbaum, "Scanning electron microscope observations of propagating acoustic waves in surface acoustic wave devices," Applied Physics Letters, vol. 37, p. 698, 1980.

[5] D. V. Roshchupkin, T. Fournier, M. Brunel, O. A. Plotitsyna, and N. G. Sorokin, "Scanning electron microscopy observation of excitation of the surface acoustic waves by the regular domain structures in the $\mathrm{LiNbO}_{3}$ crystals," Applied Physics Letters, vol. 60, p. 2330, 1992.

[6] D. Roshchupkin and M. Brunel, "Scanning electron microscopy observation of surface acoustic wave propagation in the $\mathrm{LiNbO}_{3}$ crystals with regular domain structures," IEEE Trans. Ultrasonics, Ferroelectrics \& Freq. Control, vol. 41, no. 4, pp. 512-517, 1994.

[7] D. Roshchupkin, M. Brunel, and L. Tucoulou, "Visualization of surface acoustic waves by scanning electron microscopy," Journal de Physique $I V$, vol. 04, no. C5, pp. C5-1229-C5-1232, 1994.

[8] C. Campbell, Surface Acoustic Wave Devices and their signal processing applications. San Diego, CA, USA: Academic Press, 1989.

[9] K. Hashimoto, K. Kashiwa, N. Wu, T. Omori, M. Yamaguchi, O. Takano, S. Meguro, and K. Akahane, "A laser probe based on a Sagnac interferometer with fast mechanical scan for RF surface and bulk acoustic wave devices," IEEE Trans Ultrason Ferroelectr Freq Control, vol. 58, no. 1, pp. 187-194, Jan 2011.

[10] N. C. MacDonald, G. Y. Robinson, and R. M. White, "Time resolved scanning electron microscopy and its application to bulk effect oscillators," Journal of Applied Physics, vol. 40, p. 4516, 1969.

[11] R. J. Gerdes and C. E. Wagner, "Scanning electron microscopy of resonating quartz crystals," Applied Physics Letters, vol. 18, no. 39, pp. 39-41, 1971.

[12] P. Hiesinger, "Scanning electron microscopy of resonating surface acoustic wave devices," in IEEE Proc. Ultrasonics Symposium, 1978, p. 611 .

[13] S. Sakharov, O. Buzanov, and D. Roshchupkin, "Investigation of saw and psaw propagation in lgs crystal by scanning electron microscopy method," in Proc. IEEE International Frequency Control Symposium and PDA Exhibition, 2003, pp. 698-700.

[14] E. Mackensen and L. Reindl, "Wireless passive saw identification marks and sensors," in Proceedings of the NATO Adavanced Study Institute on Smart Sensors and MEMS. Povoa de Varzim, Portugal: Springer, September 2003, pp. 155-202.

[15] D. Teyssieux, T. Baron, J.-M. Friedt, G. Martin, and P. Vairac, "Absolute phase and amplitude mapping of surface acoustic wave fields," in Proc. IEEE IFCS, Prague, Czech Rep., 2013.

[16] E. Ash and E. Paige, Eds., Rayleigh-Wave Theory and Application, 1st ed., ser. Springer Series on Wave Phenomena 2. Berlin Heidelberg: Springer-Verlag, July 1985

[17] F. Lacour, N. Courjal, M.-P. Bernal, A. Sabac, C. Bainier, and M. Spajer, "Nanostructuring lithium niobate substrates by focused ion beam milling," Optical materials, vol. 27, no. 8, pp. 1421-1425, 2005.

[18] F. Sulser, G. Poberaj, M. Koechlin, and P. Günter, "Photonic crystal structures in ion-sliced lithium niobate thin films," Optics express, vol. 17, no. 22, pp. 20291-20300, 2009.

[19] A. Gopinath and M. Hill, "Deflection beam-chopping in the sem," $J$. Phys. E: Sci. Instrum., vol. 10, p. 229, 1977.

[20] H. P. Feuerbaum and J. Otto, "Beam chopper for subnanosecond pulses in scanning electron microscopy," J. Phys. E: Sci. Instrum., vol. 11, p. $529,1978$. 